\title{
Remote Calibration of Standard Inductors
}

\author{
Yasuhiro Nakamura Member (National Metrology Institute of Japan, AIST) \\ Akihiko Yonenaga Member (National Metrology Institute of Japan, AIST) \\ Norihiko Sakamoto Non-member (National Metrology Institute of Japan, AIST) \\ Akihiko Shimoyama Non-member (Japan Electric Meters Inspection Corporation)
}

Keywords : standard, inductor, remote calibration, LCR meter, traceability

Remote calibration system for standard inductors of $10 \mathrm{mH}$ has been developed. Presently, traceable calibrations to national standards of inductance are provided to clients, only if their devices under test (DUTs), i.e. their own inductors, are transported by themselves to the National Metrology Institute of Japan (NMIJ). On the contrary, the remote calibration method enables the clients' inductors to be calibrated without being sent to the NMIJ. The "remote calibration" in this paper means that DUTs are calibrated against the transfer standards which are sent from the NMI to clients (see Fig. 1). The remote calibration is profitable for clients in some aspects; on-site calibration, maintenance of DUTs under clients' environment, elimination of uncertainty due to transportation, reduction in time and cost, and so on. We report a feasibility study on the remote calibration of standard inductors of $10 \mathrm{mH}$ at $1 \mathrm{kHz}$. (The $10 \mathrm{mH}$ at $1 \mathrm{kHz}$ is widely used as the inductance standard in industry.)

The concept of the remote calibration of DUT is shown in Fig. 1. Transfer standards that have been calibrated against the national standard in advance at the NMIJ, are sent to a client together with a coaxial scanner (switching device). The client connects DUT, i.e. its own inductor to be calibrated, a commercially-available LCR meter, and a personal computer (PC) to the scanner, and then constructs a calibration system (the remote calibration system). Client-owned LCR meter and PC are used in the system. The

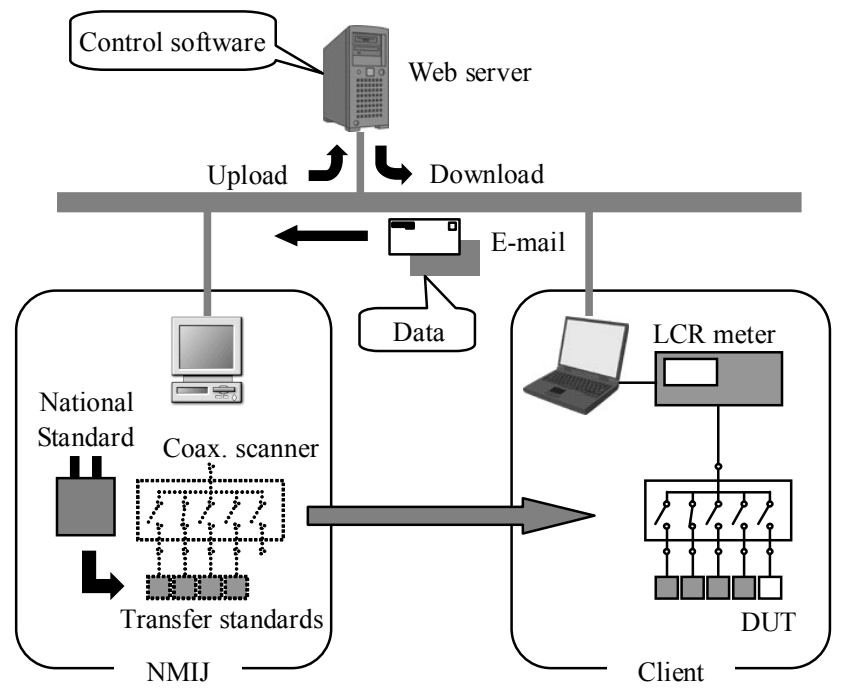

Fig. 1. Concept of the remote calibration of DUT against the national standard client starts the control software on the $\mathrm{PC}$ in order to take measurements. The control software can be downloaded by accessing to the Web server of the NMIJ. The remote calibration system is designed to be fully automatic, so that the client needs no special skill or training. After the operation of the system is completed, the obtained data automatically save on the PC. The data are sent by the client to the NMIJ by means of e-mail. The received data at the NMIJ are analyzed and the calibration result of DUT is returned by e-mail again. The calibration capability of the developed system for the remote calibration was evaluated to be the relative standard uncertainty of about 20 parts per million (ppm).

To examine the remote calibration of standard inductors, verification tests were conducted between the NMIJ and three calibration laboratories in Japan as clients. First, four transfer standards of $10 \mathrm{mH}$ were calibrated against the national standard at $1 \mathrm{kHz}$ and $23{ }^{\circ} \mathrm{C}$. Next, the coaxial scanner together with the calibrated transfer standards was carefully transported from the NMIJ to the client. Then, at the client, the measurements were carried out. The obtained measurement data were immediately transferred to the NMIJ by e-mail and analyzed.

Our own inductor was used as "DUT" in the verification tests, in order to compare the remote calibration method with the conventional method. The "DUT" used in the tests was verified in advance to be stable enough to compare two methods. The results of the verification tests are shown in Fig. 2. Marks of $\bullet, \boldsymbol{\Delta}$ and $\boldsymbol{\square}$ in Fig. 2 indicate the values obtained by the remote calibration method and the mark of $\times$ indicates the values by the conventional method. The results by two calibration methods agreed within the standard uncertainties. From the verification tests, the remote calibration of standard inductors might replace the conventional calibration in the near future.

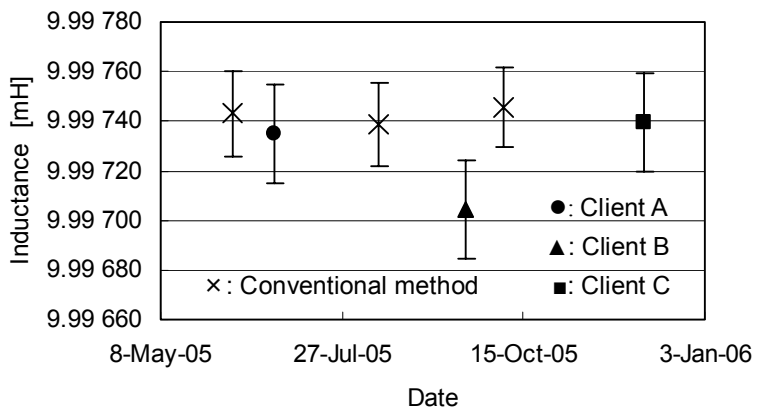

Fig. 2. Results of the verification tests 


\title{
Remote Calibration of Standard Inductors
}

\author{
Yasuhiro Nakamura* Member \\ Akihiko Yonenaga* Member \\ Norihiko Sakamoto* Non-member \\ Akihiko Shimoyama** Non-member
}

Remote calibration system for standard inductors of $10 \mathrm{mH}$ has been developed. At the National Metrology Institute of Japan (NMIJ), the calibration of inductors which is traceable to the national standards is provided to clients, only if their devices under test (DUTs), i.e. their own inductors, are carried by themselves to the NMIJ. On the contrary, the remote calibration method enables the clients' inductors to be calibrated without being carried to the NMIJ. The calibration capability of the developed system for the remote calibration was evaluated to be the relative standard uncertainty of about 20 parts per million (ppm), in the case of the calibration of $10 \mathrm{mH}$ standard inductors. From the verification test of this method for three clients in Japan, the results by the remote calibration agreed with those by the conventional method within the standard uncertainties.

Keywords : standard, inductor, remote calibration, LCR meter, traceability

\section{Introduction}

Presently, traceable calibrations to national standards are provided to clients, only if their devices under test (DUTs) are transported by themselves to national metrology institute (NMI), e.g. the National Metrology Institute of Japan (NMIJ). Therefore clients must be responsible for any accidents occurred to DUTs during their transportation, e.g. change of values of DUTs due to the ambient temperature, or the vibration of train or car.

Alternatively, the remote calibration has been presented in the last few years ${ }^{(1)}$. The "remote calibration" in this paper means that DUTs are calibrated against the transfer standards which are sent from the NMI to clients (see Fig. 1), so that clients can receive the calibration service of DUTs without transporting them to the NMI. The remote calibration is profitable for clients in some aspects; on-site calibration, maintenance of DUTs under clients' environment, elimination of uncertainty due to transportation, reduction in time and cost, and so on. Among several quantities in electrical standards, an inductance standard is taken up a subject for discussion, that is, DUTs in this paper are standard inductors of clients. We report a feasibility study on the remote calibration of standard inductors of $10 \mathrm{mH}$ at $1 \mathrm{kHz}$. (The $10 \mathrm{mH}$ at $1 \mathrm{kHz}$ is widely used as the inductance standard in industry.)

\section{Concept}

The concept of the remote calibration of DUT is shown in Fig. 1. Transfer standards that have been calibrated against the national standard in advance at the NMI, are sent to a client together with a coaxial scanner (switching device). The client connects DUT, i.e. its own inductor to be calibrated, a commercially-available LCR meter, and a personal computer (PC) to the scanner, and then constructs a calibration system (the remote calibration system). Client-owned LCR meter and PC are used in the system. The

* National Metrology Institute of Japan, AIST

Tsukuba Central 3-1, 1-1-1 Umezono, Tsukuba 305-8563

** Japan Electric Meters Inspection Corporation (JEMIC)

4-15-7, Shibaura, Minato-ku, Tokyo 108-0023

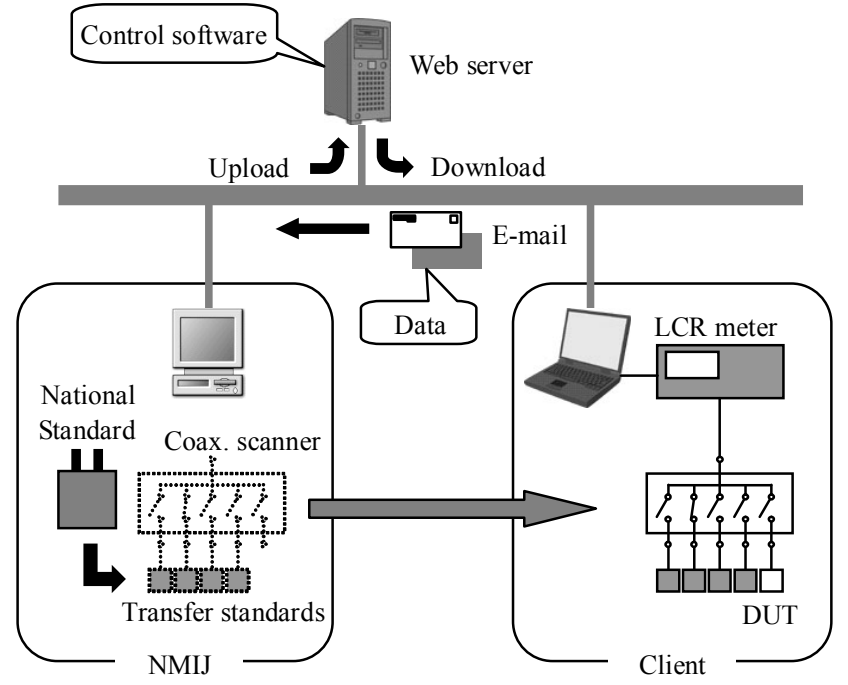

Fig. 1. Concept of the remote calibration of DUT against the national standard

client starts the control software on the PC in order to take measurements. The control software can be downloaded by accessing to the Web server of the NMI through the internet. The remote calibration system is designed to be fully automatic, so that the client needs no special skill or training. The only requirement for the client is to start the control software. After the operation of the system is completed, the obtained data automatically save on the PC. The data are sent by the client to the NMI by means of e-mail. The received data at the NMI are analyzed and the calibration result of DUT (the client's inductor) is returned by e-mail again.

\section{Calibration System}

3.1 Construction As shown in Fig. 1, the remote calibration system comprises a commercial LCR meter, a personal computer, a coaxial scanner, and four transfer standards of $10 \mathrm{mH}$. The block diagram of the system is shown in Fig. 2. The coaxial 


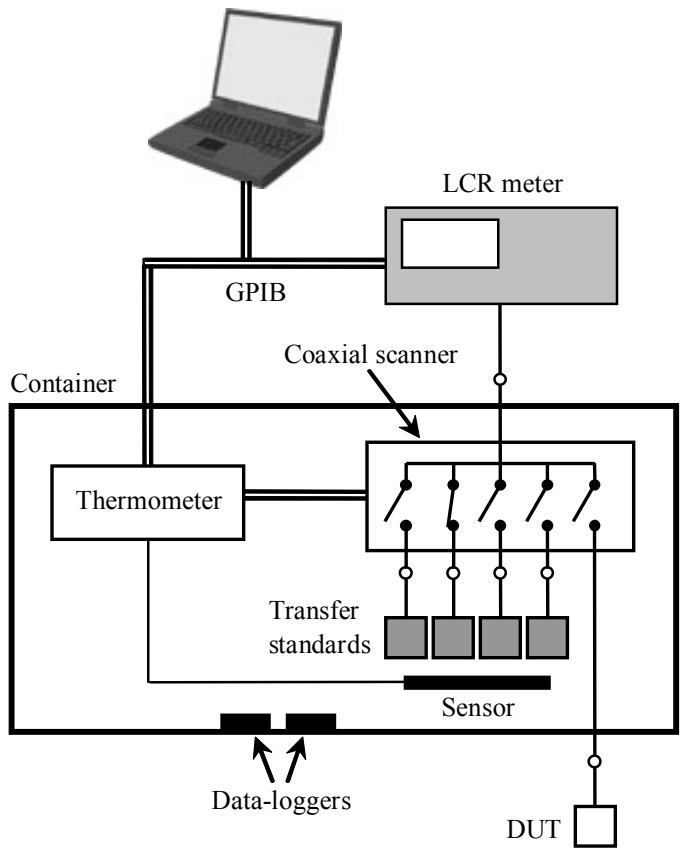

Fig. 2. Block diagram of the remote calibration system

scanner and the transfer standards are integrated into a single unit in a special container. A thermometer to measure the ambient temperature of the transfer standards is accompanied with the container. Connections between the LCR meter and the coaxial scanner, and the coaxial scanner and each inductor are made by means of a pair of four coaxial cables, although they are shown as single line in Fig. 2 for clarity. The external appearance of the remote calibration system is shown in Fig. 3. The LCR meter for the system is supposed to be a typical one widely used in industry. In this study, the type 4284A of Agilent Technologies is used as the client-owned LCR meter. The uncertainties of this type of LCR meters were previously analyzed ${ }^{(2)(3)}$. In this calibration method, the standard uncertainty due to the commercial LCR meter was estimated to be $10 \mathrm{ppm}$.

3.2 Coaxial Scanner Connections between the LCR meter and five inductors including a DUT (the client's inductor to be calibrated) are switched by using the coaxial scanner that is constructed with coaxial lead relays and a GPIB board to communicate with the PC. Usually, a coaxial relay has switches to break connections of only inner conductors of coaxial cables, not outer conductors. For high precision measurement, a coaxial relay that can make break or contact at not only inner connections but also outer connections is required. In order to switch both connections simultaneously, another lead relay to switch outer connections is added to a usual coaxial relay. Using such specially constructed relays, the scanner has been assembled. (For clarity, they are shown as simple switches in the figures.)

The scanner has five channels and the differences between each channel in inductance measurements were estimated to be less than $50 \mathrm{nH}$, which means an uncertainty of at most 5 ppm against $10 \mathrm{mH}$.

3.3 Transfer Standard Four compact-sized inductors of $10 \mathrm{mH}$ (Sunjem HLS103A; $142 \mathrm{~mm}$ in width, $88 \mathrm{~mm}$ in depth and $130 \mathrm{~mm}$ in height) are used as the transfer standards. In order to evaluate the stability of the transfer standards, their inductances were measured by using a commercially available LCR meter under the stable ambient temperature of $23 \pm 0.01{ }^{\circ} \mathrm{C}$ over ten

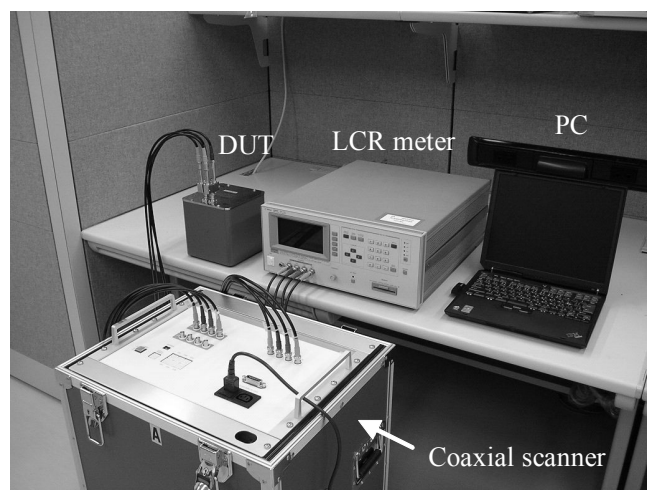

Fig. 3. External appearance of the remote calibration system

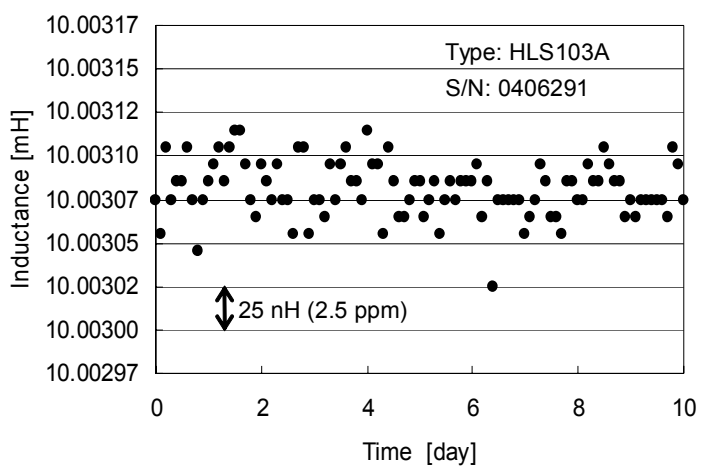

Fig. 4. Stability of one of four transfer standards

days. The fluctuations in inductance of each transfer standard were shown to be within $\pm 50 \mathrm{nH}$ of the average values for ten days. Figure 4 shows the measurement results of one of the four transfer standards. Their temperature coefficients were also measured by varying the ambient temperature from $23{ }^{\circ} \mathrm{C}$ up to $27{ }^{\circ} \mathrm{C}$, next 27 ${ }^{\circ} \mathrm{C}$ down to $19{ }^{\circ} \mathrm{C}$, and finally $19^{\circ} \mathrm{C}$ up to $23{ }^{\circ} \mathrm{C}$. The results of measurements showed that each transfer standard has the temperature coefficient of around $10 \mathrm{ppm} / \mathrm{K}$. The hysteresis in inductance due to the temperature cycling within $23 \pm 4{ }^{\circ} \mathrm{C}$ was less than $150 \mathrm{nH}$ at $23{ }^{\circ} \mathrm{C}$ for each inductor.

While the remote calibration system operates, the ambient temperature of the transfer standards in the container is measured using the thermometer, the sensor of which is set near them. Moreover, portable data-loggers are attached inside the container, in order to record the temperature and the vibration of the transfer standards during transportations between the NMIJ and the client (see Fig. 2). The transfer standards are supposed to be returned within ten days after leaving from the NMIJ.

\subsection{Control Software The LCR meter, the coaxial} scanner and the thermometer are connected through the GPIB to the PC and operated using the control software. By clicking the start button in the opening screen of the software, the whole system operates automatically and at the end of the operation, the obtained data save on the $\mathrm{PC}$ as a text format. However, the software does not run without the key code that includes some information with respect to the identification of the client, the time limit of the calibration, the measurement conditions, and so on. The clients are asked to get the key code in advance from the NMIJ.

\section{Data Analysis}

As mentioned above, at client, inductances of DUT and four 
Table 1. Uncertainty budget for the remote calibration of $10 \mathrm{mH}$ standard inductor

\begin{tabular}{|c|c|c|c|c|c|c|c|c|}
\hline \multirow{2}{*}{$\begin{array}{l}\text { Source of uncertainty } \\
\text { Transfer standards }\end{array}$} & \multirow{2}{*}{$\frac{\text { Symbol }}{u\left(L_{\text {Ave }}\right)}$} & \multirow{2}{*}{$\begin{array}{c}\text { Type } \\
\text { B }\end{array}$} & \multicolumn{2}{|c|}{$\begin{array}{c}\text { Standard uncertainty } \\
u\left(x_{i}\right)\end{array}$} & \multicolumn{2}{|c|}{$\begin{array}{l}\text { Sensitivity } \\
\text { coefficient } c_{i}\end{array}$} & \multicolumn{2}{|c|}{$\begin{array}{l}\text { Component } \\
\left|c_{i}\right| u\left(x_{i}\right)\end{array}$} \\
\hline & & & 142 & $\mathrm{nH}$ & 1 & & 142 & $\mathrm{nH}$ \\
\hline Ambient temperature at client & $u\left(T_{\text {Client }}\right)$ & B & 0.038 & $\mathrm{~K}$ & 58 & $\mathrm{nH} / \mathrm{K}$ & 2 & $\mathrm{nH}$ \\
\hline Ambient temperature at NMIJ & $u\left(T_{\mathrm{NMII}}\right)$ & B & 0.016 & $\mathrm{~K}$ & -58 & $\mathrm{nH} / \mathrm{K}$ & 1 & $\mathrm{nH}$ \\
\hline Measurements by the system & $u\left(\Delta L_{m \mathrm{Ave}}\right)$ & & 139 & $\mathrm{nH}$ & 1 & & 139 & $\mathrm{nH}$ \\
\hline \begin{tabular}{l|l} 
& Repeated measurements \\
\end{tabular} & & A & 12 & $\mathrm{nH}$ & & & & \\
\hline Stability of transfer standards & & B & 29 & $\mathrm{nH}$ & & & & \\
\hline hysteresis of transfer standards & & B & 87 & $\mathrm{nH}$ & & & & \\
\hline LCR meter & & B & 100 & $\mathrm{nH}$ & & & & \\
\hline Coaxial scanner & & B & 29 & $\mathrm{nH}$ & & & & \\
\hline \multicolumn{7}{|c|}{ Combined standard uncertainty } & 199 & $\mathrm{nH}$ \\
\hline
\end{tabular}

transfer standards are measured by using the remote calibration system. After the measurements, the obtained data are sent by e-mail to the NMIJ and analyzed.

Let the inductances at the ambient temperature of client be $L_{\mathrm{DUT}}$, $L_{m 1}, L_{m 2}, L_{m 3}$ and $L_{m 4}$, respectively. The difference in inductance between a DUT and each transfer standard is expressed by

$$
\Delta L_{m i}=L_{\text {DUT }}-L_{m i}
$$

where $i=1,2,3,4$.

Four transfer standards are calibrated against the national standard of Japan at the NMIJ in advance. However, each value of $L_{m i}$ at the client differs from the calibrated value because it has inherently a temperature coefficient and the ambient temperature at the client is different from that at the NMIJ. The temperature corrections, therefore, must be made, as in Eq. (2).

$$
L_{m i}=L_{i}+\alpha_{i} L_{0}\left(T_{\text {Client }}-T_{\mathrm{NMIJ}}\right)
$$

where $L_{i}$ is the calibrated value in terms of the national standard, $\alpha_{i}$ is the temperature coefficient of each transfer standard, $L_{0}$ is the nominal value of inductance of $10 \mathrm{mH}$ and $T$ is the ambient temperature of the transfer standards at each laboratory. From Eqs. (1) and (2), $L_{\text {DUT }}$ can be derived from four equations, as

$$
\begin{aligned}
& L_{\mathrm{DUT}}=L_{1}+\alpha_{1} L_{0}\left(T_{\text {Client }}-T_{\mathrm{NMIJ}}\right)+\Delta L_{m 1} \\
& L_{\mathrm{DUT}}=L_{2}+\alpha_{2} L_{0}\left(T_{\text {Client }}-T_{\mathrm{NMIJ}}\right)+\Delta L_{m 2} \\
& L_{\mathrm{DUT}}=L_{3}+\alpha_{3} L_{0}\left(T_{\text {Client }}-T_{\mathrm{NMIJ}}\right)+\Delta L_{m 3} \\
& L_{\mathrm{DUT}}=L_{4}+\alpha_{4} L_{0}\left(T_{\text {Client }}-T_{\mathrm{NMIJ}}\right)+\Delta L_{m 4}
\end{aligned}
$$

Then, by averaging four equations, the inductance of the DUT can be given as,

$$
L_{\text {DUT }}=L_{\text {Ave }}+\alpha_{\text {Ave }} L_{0}\left(T_{\text {Client }}-T_{\text {NMIJ }}\right)+\Delta L_{m \text { Ave }}
$$

where

$$
\begin{aligned}
& L_{\text {Ave }}=\frac{L_{1}+L_{2}+L_{3}+L_{4}}{4} \ldots \ldots \ldots \ldots \ldots \ldots \ldots \ldots \ldots \ldots \ldots \ldots \ldots \ldots \ldots \ldots \ldots \ldots \ldots \\
& \alpha_{\text {Ave }}=\frac{\alpha_{1}+\alpha_{2}+\alpha_{3}+\alpha_{4}}{4} \ldots \ldots \ldots \ldots \ldots \ldots \\
& \Delta L_{m \text { Ave }}=\frac{\Delta L_{m 1}+\Delta L_{m 2}+\Delta L_{m 3}+\Delta L_{m 4}}{4}
\end{aligned}
$$

Moreover, with the reference to "Guide to the Expression of

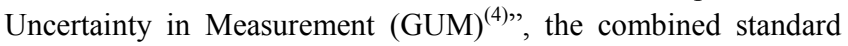
uncertainty of measurement, $u_{\mathrm{c}}\left(L_{\mathrm{DUT}}\right)$ can be espressed as,

$$
\begin{aligned}
u_{c}^{2}\left(L_{\mathrm{DUT}}\right) & \left(\frac{\partial L_{\mathrm{DUT}}}{\partial L_{\text {Ave }}}\right)^{2} u^{2}\left(L_{\text {Ave }}\right)+\left(\frac{\partial L_{\mathrm{DUT}}}{\partial \alpha_{\text {Ave }}}\right)^{2} u^{2}\left(\alpha_{\text {Ave }}\right) \\
& +\left(\frac{\partial L_{\mathrm{DUT}}}{\partial T_{\text {Client }}}\right)^{2} u^{2}\left(T_{\text {Client }}\right)+\left(\frac{\partial L_{\mathrm{DUT}}}{\partial T_{\mathrm{NMIJ}}}\right)^{2} u^{2}\left(T_{\mathrm{NMIJ}}\right) \\
& +\left(\frac{\partial L_{\mathrm{DUT}}}{\partial \Delta L_{m \text { Ave }}}\right)^{2} u^{2}\left(\Delta L_{m \mathrm{Ave}}\right) \\
= & u^{2}\left(L_{\text {Ave }}\right)+L_{0}^{2}\left(T_{\text {Client }}-T_{\mathrm{NMIJ}}\right)^{2} u^{2}\left(\alpha_{\text {Ave }}\right) \\
& +\left(\alpha_{\text {Ave }} L_{0}\right)^{2} u^{2}\left(T_{\text {Client }}\right)+\left(-\alpha_{\text {Ave }} L_{0}\right)^{2} u^{2}\left(T_{\mathrm{NMIJ}}\right)+u^{2}\left(\Delta L_{m \text { Ave }}\right)
\end{aligned}
$$

From the evaluations of the system described in Sec. 3, the sources of uncertainties in the remote calibration of $10 \mathrm{mH}$ inductor are summarized in Table 1. On the basis of Eq. (11), the combined standard uncertainty of the remote calibration, $u_{\mathrm{c}}\left(L_{\mathrm{DUT}}\right)$ was estimated to be $199 \mathrm{nH}$, that is, about $20 \mathrm{ppm}$ against $10 \mathrm{mH}$, which is the same level of uncertainty as that in the present service provided by the NMIJ. The uncertainty due to the temperature coefficient, $\left|L_{0}\left(T_{\text {Client }}-T_{\text {NMIJ }}\right)\right| u\left(\alpha_{\text {Ave }}\right)$ is not shown in Table 1, because it is negligible.

\section{Verification Test}

To examine the remote calibration of standard inductors, verification tests using the developed system were conducted between the NMIJ and three calibration laboratories in Japan as clients. First, four transfer standards of $10 \mathrm{mH}$ were calibrated against the national standard of inductance at $1 \mathrm{kHz}$ and $23{ }^{\circ} \mathrm{C}$. Next, the container that included the coaxial scanner and the calibrated transfer standards was carefully transported from the NMIJ to the client. Special transportations of the container were made under the condition of almost stable in ambient temperature to be $23 \pm 2{ }^{\circ} \mathrm{C}$. During transportations, changes of the ambient temperature and the vibration of the container were recorded by the portable data-loggers, in order to check whether the transfer standards had not been damaged by temperature change, or shock. At the client, the remote calibration system was constructed with the container, the client-owned LCR meter, the PC, and "DUT (the client's inductor to be calibrated)". Then, the measurements were carried out by the client. The obtained measurement data were immediately transferred to the NMIJ by e-mail and analyzed at the NMIJ.

Actually, our own inductor was used as "DUT" in the verification tests, in order to compare the remote calibration method with the conventional method, or to compare the results of 


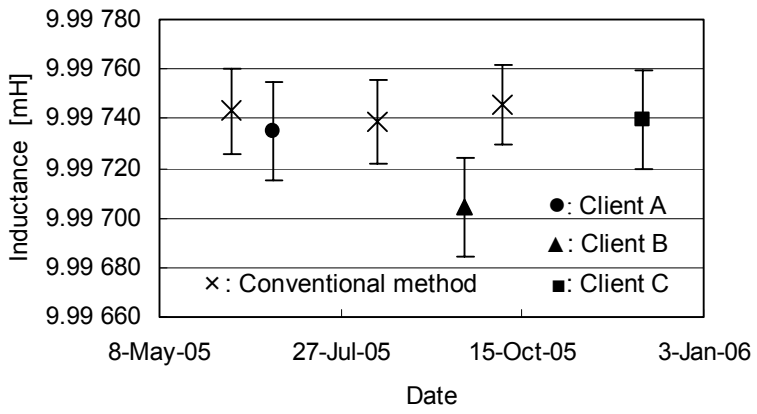

Fig. 5. Results of the verification tests (DUT is the NMIJ-owned inductor of S/N 0202)

tests at three clients each other. Our own inductor used in the tests was verified in advance to be stable enough to compare two methods, or to compare inter-clients. The results of the verification tests are shown in Fig. 5. Marks of $\bullet, \boldsymbol{\Delta}$ and $\boldsymbol{\square}$ in Fig. 5 indicate the values obtained by the remote calibration method and the mark of $\times$ indicates the values by the conventional method. Although the value obtained at Client $\mathrm{B}$ differs from others, the results by two calibration methods agrees within the standard uncertainties. (We have found that the cause of the difference at Client B was due to the connections between the transfer standards and the coaxial scanner being loosened by vibration during the transportation.) From the verification tests, the remote calibration of standard inductors might replace the conventional calibration in the near future.

\section{Conclusion}

The remote calibration method of standard inductors was presented. Actually, the remote calibration system has been developed in order to calibrate the client's inductor of $10 \mathrm{mH}$ without its being sent to the NMIJ. The standard uncertainty of the system was evaluated to be $199 \mathrm{nH}$, that is, the relative standard uncertainty be about $20 \mathrm{ppm}$. From the verification tests, the calibrated values by the remote calibration method agreed with the values obtained by the conventional method within the standard uncertainties.

\section{Acknowledgement}

The authors would like to thank S. Matsuzawa and K. Hanaoka of Nagano Prefecture General Industrial Technology Center, T. Emori and S. Miyajima of Murata Manufacturing Co., Ltd. and S. Sakagami of JEMIC for cooperating on the verification tests.

(Manuscript received Feb. 24, 2006, revised May 19, 2006)

\section{References}

(1) S. A. Awan, J. M. Williams, S. Bryant, and P. C. A. Roberts : "Evaluation of the 4950 Multifunction Transfer Standard for Internet-based Calibration of Electrical Quantities," Proc. 2001 NCSL International Workshop \& Symposium (2001)
(2) A. Yonenaga and Y. Nakamura : "Simple Inductance Measurement Method Using a Commercial LCR Meter", IEEJ Trans. FM, Vol.125, No.6, pp.544-548 (2005-6)

(3) A. Yonenaga and Y. Nakamura : "Uncertainty Evaluation of Inductance Measurement by Substitution Method Using an LCR Meter", 2005 National Convention Record IEE Japan, No.1-127, pp.156-157 (2005) (in Japanese)

(4) Guide to the Expression of Uncertainty in Measurement, International Organization for Standardization (ISO), Geneva (1995)

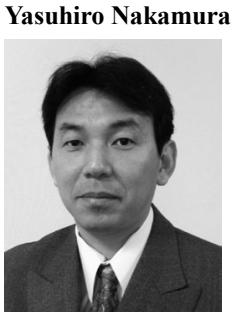

(Member) was born in Ise, Mie prefecture, Japan, in 1965. He received the B.S., M.S., and Ph.D. degrees in Electrical Engineering from Doshisha University, Kyoto, Japan, 1989, 1991, and 1994, respectively. He joined the Electrotechnical Laboratory, Tsukuba in 1994 and transferred to the National Metrology Institute of Japan, AIST in 2001. He has worked mainly in the field of impedance standards, such as capacitance standard and inductance standard. His current interests include precision measurements, and dc and low frequency electricity standards.

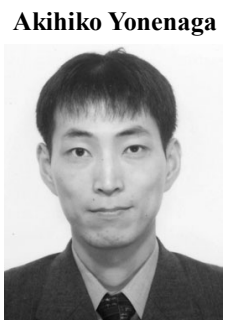

(Member) was born in Nishinomiya, Japan, on July 19, 1973. He received the B.E. and the M.E. degrees from University of Tokyo in 1996 and 1998, respectively. He is presently a research scientist at National Metrology Institute of Japan, National Institute of Advanced Industrial Science and Technology (NMIJ/AIST). He has worked on research of the pressure standards and inductance standards. He is a member of the Society of Instrument and Control Engineers.

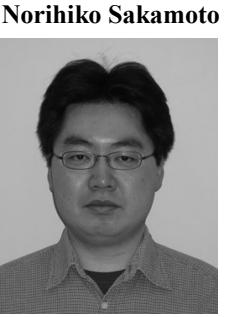

(Non-member) was born in Saitama prefecture, Japan, in 1975 . He received the B. E. degree in surface science from Tokyo University of Science in 1999 and received the M. E. and the Ph. D. degrees in metal-oxide physics and chemistry from Tokyo Institute of Technology in 2001 and 2005, respectively. He joined the National Metrology Institute of Japan, National Institute of Advanced Industrial Science and Technology (NMIJ/AIST), Tsukuba, in 2005. He engages in research of capacitance and AC voltage ratio standards.

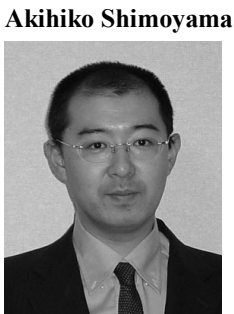

(Non-member) was born in Kumamoto, Japan, in 1970. He received the B. E. and the M. E. degrees from Saga University in 1993 and 1995, respectively. He joined the Japan Electric Meters Inspection Corporation (JEMIC), Tokyo, in 1995. He has been engaging in the precision measurements relating to the low frequency impedance standards, such as capacitance standards and inductance standards. 